\title{
BMJ Doctors accessing mental-health Open services: an exploratory study
}

\author{
Josephine Stanton, ${ }^{1}$ Patte Randal ${ }^{2}$
}

To cite: Stanton J, Randal P. Doctors accessing mental-health services: an exploratory study. BMJ Open 2011:1:e000017.

doi:10.1136/

bmjopen-2010-000017

- Prepublication history for this paper is available online. To view these files please visit the journal online (http:// bmjopen.bmj.com).

Received 3 November 2010 Accepted 2 February 2011

This final article is available for use under the terms of the Creative Commons Attribution Non-Commercial 2.0 Licence; see http://bmjopen.bmj.com

${ }^{1}$ Child and Family Unit, Auckland Healthcare, Auckland Mail Centre, Auckland, New Zealand ${ }^{2}$ Rehabilitation Psychiatry, Buchanan Rehabilitation Centre, Auckland Healthcare, Auckland Mail Centre, Auckland, New Zealand

Correspondence to Dr Josephine Stanton; josephines@adhb.govt.nz

\section{ABSTRACT}

Objective: To develop a more in-depth understanding of how doctors do and do not access mental healthcare from the perspectives of doctors themselves and people they have contact with through the process.

Design: Qualitative methodology was used with semistructured interviews transcribed and analysed using Grounded Theory. Participants were 11 doctors with experience as patients of psychiatrists, four doctor and four non-doctor personal contacts (friends, family and colleagues) and eight treating psychiatrists.

Results: Participants described experiencing unrealistic expectations and a harsh work environment with poor self care and denial and minimisation of signs of mental health difficulties. Doctor contacts described particular difficulty in responding effectively to doctor friends, family and colleagues in need of mental healthcare. In contrast, non-doctor personal contacts were more able to identify and speak about concerns but not necessarily to enable accessing adequate mental-health services.

Conclusions: Three areas with potential to address in supporting doctors' accessing of appropriate healthcare have been identified: (1) processes to enable doctors to maintain high standards of functioning with less use of minimisation and denial; (2) improving the quality and effectiveness of informal doctor-to-doctor conversations about mental-health issues among themselves; (3) role of non-doctor support people in identifying doctors' mental-health needs and enabling their access to mental healthcare. Further research in all these areas has the potential to contribute to improving doctors' access to appropriate mental healthcare and may be of value for the general population.

\section{INTRODUCTION}

Living in a culture where doctors are seen as healthy people who treat sick patients creates a paradox for a doctor moving into a patient role. Accordingly, many doctors do not make use of usual channels for accessing healthcare $^{1}$ and continue to treat themselves despite guidelines to the contrary. ${ }^{2}$ Much expert opinion about barriers to healthcare for doctors is available in the literature, but the quality of the data available is limited. ${ }^{3}$

Moving into the role of a patient with psychological illness has been described as

\section{ARTICLE SUMMARY}

\section{Article focus}

- Doctors' accessment of adequate mental healthcare is less than optimal.

- Family and community contacts have an important role in accessing mental healthcare.

- Our understanding of the processes related to doctors accessing mental healthcare can be improved by exploring perspectives of doctor patients, their support people and treating psychiatrists.

\section{Key messages}

- Doctors' unrealistic expectations of themselves and associated minimisation and denial of a range of self care needs may function as a barrier to accessing mental healthcare.

- Addressing how doctors respond to other doctors in informal conversations indicating mental healthcare needs may be helpful in improving access to care.

- Non-doctor support people may have a valuable role in enabling doctors to access appropriate mental healthcare.

- All these areas need further research.

Strengths and limitations of this study

- This is the first study of its kind and generates new insights in an important area.

- Because of challenges in recruiting doctors with experience as patients of psychiatrists, a hard-toreach group, the sample is small and not broadly representative.

particularly challenging. ${ }^{4}$ There is increasing concern about doctors' mental health and effectiveness in accessing mental-health services. $^{5-7}$

Out of this concern, a literature documenting, researching and recommending interventions for medical students and doctors is emerging. ${ }^{8-11}$ The research base is limited. Individual case information has been made available. ${ }^{12}{ }^{13}$ Questionnaire surveys provide important information about attitudes, ${ }^{14} 15$ but the depth and complexity they are able to contribute to our understanding are limited. We need more understanding about how doctors do and do not access 
mental healthcare. This is likely to be helpful in addressing barriers to healthcare for doctors. Moreover, Kay $e t a l^{3}$ point out similarities between doctors and the general population in accessing mental healthcare. Thus, increasing understanding of barriers for doctors accessing mental healthcare may have implications for the wider population. Likewise, research in the general population has demonstrated the importance of family and community contacts in facilitating access to mentalhealth services. ${ }^{16}$ So, we need more understanding of how this may also be important for doctors.

The aim of this paper is to develop a more in-depth understanding of how doctors do and do not access mental healthcare from the perspectives of doctors themselves and people they have contact with through the process.

The data presented in this paper are drawn from a more wide-ranging multiperspective qualitative study on doctors as patients of psychiatrists.

\section{Participants}

Recruitment was challenging. We sought informationrich participants, doctors with experience as a patient of a psychiatrist who were fluent in English and had capacity to consent. We approached seven doctors who were known to one or other of the researchers through personal or collegial contact as having had experience as patients of psychiatrists. Five agreed to participate. Formal channels such as the Medical Council and support providers to doctors declined to participate because of concerns about confidentiality. One of the researchers (PR) had been involved in developing an informal support network for doctors with mental-health issues, and three potential participants were identified via this role. Two agreed to participate. She also put out an invitation to participate in the study to members of a currently developing local internet site for peer support for doctors. One member specifically declined, and there were no other responses. Two were identified and referred to the researchers by other participants. One self-identified to the researchers following listening to a presentation of preliminary data. One was referred by a treating psychiatrist.

Eleven doctors, five men and six women, were interviewed. The age range was 32-54. The number of years since graduation was $5-28$ years. Diagnoses identified by the doctors themselves were Depression, Psychotic Depression, Bipolar Disorder, Generalised Anxiety Disorder, Brief Psychotic Episodes, Bipolar disorder with a differential diagnosis of Schizophrenia, Borderline traits and Poly Substance Dependence.

Nine were working in clinical medicine at the time of the interview, five as specialists. Six were working in psychiatry, two as specialist psychiatrists and four as generalists with a special interest in psychiatry. One was working in General Practice. At the time of initial identification of mental-health needs, only two were working in psychiatry. Six were working in General Practice, two were in specialist training positions, one was a house surgeon, one was a student, and one was a specialist. The range of time off work owing to illness was up to 2 years.

Psychiatrists with experience of treating doctors as patients were recruited separately. Eleven were approached. They were selected by the researchers to provide a range of orientations and practice type. Three declined interview. Eight were interviewed. The range of years since qualification as a psychiatrist was $12-39$ years. Estimated numbers of doctors treated ranged from 8-12 to $60-70$. All had private-practice experience, and most, but not all, of their experience of treating doctor-patients had been in the private sector.

Eight contact people (friends, family or colleagues) were identified by the doctors with experience as patients. Four of these were themselves doctors, two of whom had also been recruited as doctors with experience as patients of psychiatrists. In total, 25 participants were interviewed.

\section{Procedure}

Participants were interviewed individually by the two researchers together for one or two interviews of up to $90 \mathrm{~min}$ each. The interviews were initially open. The participant was invited to choose a place to begin. Most told their story. This part of the interview was not timelimited, and a second interview was scheduled when needed. Participants were encouraged to range broadly over their experience, with prompts such as, 'Can you tell us more about that?' Questioning in the interview was focused towards bringing forward the experiences, thoughts, values, etc of the participant-for example, 'How did you decide to ...?,' 'How did you experience/ understand that?,' 'What were your hopes/fears when you ....?'

A tentative checklist of relevant issues was developed initially from published personal accounts, personal and clinical contact with doctors with mental-health issues, and seminars for psychiatrists treating doctors. This was revised and developed in accord with ongoing data analysis of interviews. Most issues were covered in the open part of the interview. If not, they were specifically enquired about. Issues from the checklist relevant to this paper were identifying and managing vulnerability, other people knowing, identifying mental illness (what helped and hindered this?), decision to access care (how was this made?), finding/choosing a psychiatrist, talking to colleagues, friends, family, etc, stigma, and worry about and experience of the Medical Council.

Ethical approval was obtained from the Ministry of Health Ethics Committee (No AKY/04/12/344).

\section{Data analysis}

The interviews were transcribed verbatim from recordings by a typist and reviewed by one of the researchers (JS). Identifying data were removed. A grounded-theory approach to data analysis was used. ${ }^{17} 18$ The transcripts were closely read by each of the researchers individually and independently coded using the Qualitative Solutions and Research NVIVO computer software (QSR 
International, Doncaster, Australia). The two sets of codes and themes were then extensively and continuously discussed and compared. Convergence and divergence in accounts were both explicitly sought, with particular emphasis on exceptions. Emerging themes and higherlevel codes were fed into the ongoing interviewing process. This process was repeated, at times in part and at times in whole, through the data-collection process. In returning to the transcripts, the recoding was focused on both confirmation and disconfirmation of hypotheses, and an evolving analysis of themes. Additional depth to this process was contributed by comparing and contrasting accounts from different groups of participants.

\section{Researchers}

Both researchers are doctors with decades of experience working in psychiatry across a range of disorders and service types. One of the researchers has personal experience of psychosis and depression (PR). Both researchers have experience as network members of doctors engaging as patients of psychiatrists and limited experience in engaging with doctors as patients. One of the researchers is working as a child and adolescent psychiatrist, using biological as well as psychological approaches and compulsory care (JS). The other researcher is working psychologically with people with severe and chronic psychiatric disorder (PR). Both are committed to working creatively and reflectively, developing strategies for empowering and enabling people who are using mental-health services ${ }^{19}$ (http://www. collaborativepsychiatry.com).

\section{RESULTS}

Accounts of male and female participants did not generate differences in coding. Where an indication of gender is used in presenting the data, these are assigned randomly, irrespective of the gender of the person making the comment.

\section{Doctors as super people}

All of the doctor participants described elements of a culture of unrealistic expectations of themselves and one another, needing to 'know everything about everything' and not able to make 'any mistakes.'

you have a particular way that you think that doctors are meant to act ... be calm, cool and collected and ... having everything together. ... dealing with life and death and being in control. ... putting yourself on the back burner and just fighting for your patients.

To show vulnerability was to risk losing respect of peers and seniors. Some described having empathy for a patient in distress being associated with the risk that you might 'reveal your own emotions and vulnerability.'

Participants described some variability in the whole heartedness with which they took up this idea. One described thinking it was, 'Ok to be vulnerable' but you 'did not show it.' Another described awareness of a discrepancy between the expectation that they be 'kind, caring and compassionate' towards other people but 'not to have that for yourself.' Another described how medical students 'tried to be accepting' when a class member developed a psychotic illness. One described awareness of these expectations but not having a sense, himself, of patients needing to see him as invulnerable.

\section{Pressure in the workplace}

Bullying and lack of emotional safety were described in the workplace, particularly in years following graduation but continuing through later practice.

there was that absolute sense of 'can't cope, sling your hook, off you go, you're obviously not made for it, you're obviously not good enough'

Treating psychiatrists spoke of competitiveness, criticism and harshness among colleagues in medicine from their own experience and listening to other doctors.

Most doctor participants described not taking care of basic needs such as for sleep, or even a coffee break. Some described becoming more able to do this over time.

\section{Maintaining the myth}

Some of the participants spoke about how difficult it was to maintain the myth of being a super-person and the cost of this.

I've got this dichotomous view of myself; at one level I do think I'm very careful and I do generally deal quite well with patients. [um] But on the other hand if there's any hint of criticism I suddenly think I'm no good.

\section{Failing to identify signs of illness}

Several participants described actively denying and minimising awareness of difficulties and early signs of illness.

I managed to keep convincing myself that I was quite capable and I managed to convince a lot of other people [doctors]

The commonest response to a perceived difficulty in functioning was to just 'keep going.'

I'd be dragging myself around. I remember at the end of the day I had to lie down for about an hour before I could drive home.

\section{Initiating help-seeking}

Even once the recognition of need for help was acknowledged, the decision to see a psychiatrist was a last resort for most participants. Several described selftreating with medication, exercise, relaxation, etc until a point of crisis or desperation was reached.

It was just getting so bad, like I was just, things were becoming so unmanageable in my life. 
Doctor-contact people - friends, colleagues and family who were also doctors

Six of the eight treating psychiatrists and five of the 11 doctor patients described experiences as doctor-contact people in addition to the four doctors identified as contact people. Thus, 15 of the 21 participants who were doctors described experience as contacts with doctors with mental-health issues. They were in a range of roles including friend, partner, colleague or supervisor. The coding of their descriptions of experiences in all these roles overlapped substantially and therefore were analysed together. There was little overlap with the descriptions of the 4 non-doctors identified as personal contacts.

Some of the doctors described difficulty in perceiving need for care, 'particularly mental health' in doctor friends and colleagues with a tendency to over or underestimate the seriousness of issues. One participant described being approached for a prescription for benzodiazapines and feeling 'off-guard,' 'completely dis-empowered.'

wanting to be helpful but ... I felt so much on the defensive being asked for something and not delivering it that I didn't sort of think, think more broadly ... obviously she may be in some sort of trouble

Another described failing to respond usefully to a phone call from a colleague asking for information around treatment of depression.

I didn't hear the asking for help in it. ... I heard his uncomfortableness. ... how I dealt with the uncomfortableness was to draw away, you know, rather than to step in. And again it was this fear of stepping in where I wasn't wanted, of his feeling humiliated because of me finding out about his vulnerability and the fact that he was a mess.

Several doctors described identifying signs of illness in friends or colleagues and not speaking about them.

I can pick up on an intuitive level that they [other doctors] are depressed and I struggle to know what to do about it because you can't sort of say, 'I've been there and I can see that you're struggling, do you want to have a little talk to me?'

Participants described finding it hard to know 'how much to probe' in a context which is 'sort of professional' and 'more a social [conversation].'

the sense of paralysis around 'what the hell do I do?' and being tied up with just the complexity of it all.

Some of the doctor contacts described regret at having responded to hearing experiences described by a doctor friend in terms of symptoms rather than an example of a range of ordinary experience.

As a friend who wasn't trained you wouldn't see it as a symptom, you'd just go 'that's $[\mathrm{x}]$.'
Some of the doctor patients described how unhelpful it had been when doctor friends had responded to them by identifying symptoms or recommending mental health services rather than hearing and responding as to an ordinary human concern.

One doctor spouse described concern that her being a doctor delayed rather than facilitated access to care as both she and doctor colleagues avoided accessing 'to protect his identity and his career.'

Anyone else would have dialed 111 and the ambulance would have been there and he would have been admitted

In contrast to the above accounts, one of the doctor colleagues who had felt unable to be helpful was described by the doctor patient as having been 'enormously' helpful in seeing the size of the problem and 'putting the flag up.'

Several of the doctor-contact people described feeling they had learnt from these and other experiences and would feel more able to respond effectively in the future.

Non-doctor-contact people: friends, colleagues and family who were not doctors

Non-medical network members described identifying difficulties and the need for help in their partners but not necessarily identifying these as mental illness.

I was aware that she was a bit down and that things weren't right, you know. The Wordsworth poem; 'Some natural sorrow, loss, or pain, that has been, and will be again.' It felt like that kind of situation to me.

Another described being aware of her partner being 'grumpy' and knowing that something needed to happen but not having the knowledge to identify depression. Their personal knowledge was overshadowed by the medical knowledge of their partners leaving them disempowered in this context.

[Her being a doctor] just made it very hard for me to convince her... um... that she might need help because she's the expert.

Some described feeling that their loved one's concern about confidentiality impeded accessing care. Some of those who did take an active role in calling a crisis team or ambulance described difficulty getting an adequate response and then facing their partner's anger and rejection of what was offered.

\section{Treating psychiatrists}

Treating psychiatrists described delays in doctors accessing treatment. They described most doctor patients as self-referring, some sent by their employer and some by the Medical Council. Referral via general practitioner was less usual. They described doctors selfreferring in response to concern about their work performance and associated fear of complaints to the Medical Council rather than presenting as having a mental illness. 


\section{DISCUSSION}

\section{Summary of main findings}

1. Doctors described having a culture of unrealistic expectations of themselves and each other.

2. These expectations were associated with denial and minimisation of need for self care, vulnerability and early signs of illness. In this way, they formed a barrier to help-seeking.

3. Doctor colleagues, friends and partners, whether recruited as doctor patients, doctor contacts or treating psychiatrists, described experiences of considerable difficulty in identifying concerns and speaking about these to other doctors.

4. Non-doctor personal contacts described being able to identify difficulties and speak about them but not necessarily to enable effective help-seeking for the doctor.

\section{Strengths and limitations of study}

This is an in-depth study of an information-rich group in an area where more understanding is acutely needed. Including the perspectives of the doctors themselves as well as doctors and non-doctors with whom they had contact in the process has given us the opportunity to generate new insights.

Challenges in recruiting have meant there is a selfselection bias. Most participants were recruited via personal knowledge or recommendation. In the current climate, this personal knowledge may be necessary to create the safety for agreement to participate.

Personal knowledge based on prior relationship with some of the participants by at least one of the researchers, as well as the openly stated personal experience of being a patient of a psychiatrist of one of the researchers (PR), has the potential strength of facilitating trust and enabling openness on the part of the participants and also of increasing depth of understanding for the researchers.

Conversely, there is also a potential limitation that the preconceived understandings, attitudes or values inherent in this personal knowledge could interfere unhelpfully in the process of engagement with the data. It should be noted that only one of the participants was well known to both the researchers, and only one of the researchers (PR) had personal experience of being a patient of a psychiatrist. Ongoing comparison between the independent data analyses undertaken by each researcher was used to optimise the potential usefulness of differences in personal knowledge in enriching the level of engagement with the data.

Doctors working in psychiatry are over-represented, and doctors currently working in General Practice under-represented. The small number of non-doctor personal contact people is of particular significance, as the study indicated a marked difference in the contribution of doctor and non-doctor-contact people to doctors accessing mental-health services. This has not previously been identified.
Relationship of findings to previous research

The culture of unrealistic expectations of doctors described here has been previously identified. ${ }^{5} 1220$ Clearly doctors cannot know everything, cannot be infallible and will have a range of health issues. Self-perceived medical errors are associated with distress and a range of negative outcomes for physicians. ${ }^{21}$ Unless doctors are able to acknowledge realistic limitations and vulnerability, they will need to use denial to protect themselves from conscious awareness of their vulnerability and fallibility, and minimise these to others. Denial and minimisation have been identified in doctors. ${ }^{22}{ }^{23}$ Potential adverse effects of denial of fallibility were demonstrated by $\mathrm{Wu}$ et al. ${ }^{24}$ They found that doctors who took responsibility for making an error experienced more distress but were more likely to engage in constructive remedial processes. The limited role of intrapersonal denial as a barrier to accessing mental healthcare is demonstrated by Tyssen et $a l,{ }^{25}$ in that most of the doctors in their study who described themselves as having had mental-health problems in the previous year had not sought help for them.

Informal conversations with colleagues have been identified as one of the ways doctors access healthcare. $^{26} 27$ The difficulty described in this study by doctor friends, colleagues and partners in identifying and speaking about mental-health issues in this context has not previously been demonstrated in research. That this may be a more widespread phenomenon is indicated by Thompson et $a l \mathrm{~s}^{4}$ finding that GPs played down evidence of colleagues being physically unwell.

There is some research into doctors' descriptions of how they would respond to hypothetical scenarios. Farber et $a l^{28}$ found physicians were more likely to say they would report a physician in a hypothetical scenario who was impaired owing to substance abuse than one who was psychologically or cognitively impaired. The tendency to report was associated with valuing protecting the good of society over the rights of the individual. Roberts $e t a l^{29}$ found that medical students described valuing of confidentiality over accessing appropriate help in responses they described to vignettes of medical students with serious symptoms and functional impairment. There were no significant differences in described responses to mental illness, substance abuse or diabetes.

The idea that non-doctor personal contact people are more able to identify and speak about mental-health issues in doctors has not been previously reported. A relationship with a supportive spouse has been described as being protective with respect to risk of burnout ${ }^{30} 31$ and doctors have described themselves as more likely to disclose a mental-health issue to a non-doctor contact than a professional. ${ }^{15}$

\section{CONCLUSIONS}

This study indicates three important areas to address and research further in improving access to mental healthcare for doctors. The first is that of managing unrealistic expectations in order to support doctors to 
maintain high standards of functioning and to acknowledge vulnerability and fallibility to selves and others in appropriate ways.

The second is that of informal doctor to doctor conversations. It may be that improving the quality and effectiveness of informal doctor-to-doctor conversations is an area where a difference can be made in improving doctors' access to mental-health services. This may also be of significant benefit for doctors' general health.

The third area is the potential role of non-doctor contact and support people in being more readily able, than other doctors, to identify and speak about mentalhealth issues with doctors.

These findings could also be important for other groups and the general population.

Funding The research was funded by the Oakley Research Foundation, an independent trust.

Competing interests None.

Ethics approval Ethics approval was granted by the Ministry of Health Ethics Committee (No AKY/04/12/344) and consent forms approved them were signed by all participants.

Provenance and peer review Not commissioned; externally peer reviewed.

\section{REFERENCES}

1. Tyssen R. Health problems and the use of health services among physicians: a review article with particular emphasis on Norwegian studies. Ind Health 2007;45:599-610

2. Forsythe M, Calnan M, Wall B. Doctors as patients: postal survey examining consultants and general practitioners' adherence to guidelines. BMJ 1999;319:605-8.

3. Kay M, Mitchell G, Clavarino A, et al. Doctors as patients: a systematic review of doctors' health access and the barriers they experience. Br J Gen Pract 2008;58:501-8.

4. Thompson WT, Cupples ME, Sibbett $\mathrm{CH}$, et al. Challenge of culture, conscience, and contract to general practioners' care of their own health: qualitative study. BMJ 2001;323:728-31.

5. Department of Health. Mental health and ill health in doctors, 2008. http://www.dh.gov.uk/prod_consum_dh/groups/dh_digitalassets/ @dh/@en/documents/digitalasset/dh 083090.pdf 18.

6. Houston D. Psychological distress and error making among junior house officers. Br J Health Psychol 1997;2:141-51.

7. Taylor C, Graham J, Potts $\mathrm{H}$, et al. Impact of hospital consultants poor mental health on patient care on patient care. $\mathrm{Br} J$ Psychiatry 2007;190:268-9.

8. Amy I, Kirsty F. The competent novice: how to handle stress and look after your mental health. BMJ 2009;338:1139-44.

9. Krasner M, Beckman H, Suchman A, et al. Association of an educational programme in mindful communcation with burnout, empathy, and attitudes among primary care physicians. JAMA 2009;302:1284-93.

10. Shanafelt TD. Enhancing meaning in work: a prescription for preventing physician burnout and promoting patient-centred care. JAMA 2009;302:1338-40.

11. Isaksson KE, Gude T, Tyssen R. Counselling for burnout in Norwegian doctors: ond year cohort study. BMJ 2004;337:a2004.

12. Jones P. Doctors as Patients. Oxford: Radcliffe Publishing, 2005.

13. Emson D. Daksha emson. BMJ 2004;328:291.

14. Mukherjee $R$, Wijetunge $M$, Surgenor $T$. The stigmatisation of psychiatric illness: the attitudes of medical students and doctors in a London teaching hospital. Psychiat Bull 2002;26:178-81.

15. Hassan TM, Ahmed SO, White AC, et al. A postal survey of doctors attitudes to becoming mentally ill. Clin Med 2009;9:327-32.

16. Lincoln C, Harrigan S, McGorry PD. Understanding the topography of the early psychosis pathways. An opportunity to reduce delays in treatment. Br J Psychiatry Suppl 1998;172:21-5.

17. Glaser BG, Strauss AL. The Discovery of Grounded Theory: Strategies for Qualitative Research. Chicago: Aldine Publishing Company, 1967.

18. Strauss A, Corbin J. Basics of Qualitative Research: Techniques and Procedures for Developing Grounded Theory. Thousand Oaks, CA: Sage, 1998.

19. Randal P, Stewart M, Proverbs D, et al. 'The Re-covery Model' An integrative developmental stress-vulnerability-strengths approach to mental health. Psychosis 2009;1:122-33.

20. Ingstad B, Christie VM. Encounters with illness: the perspective of the sick doctor. Anthropol Med 2001;8:201-10.

21. West CP, Huschka MM, Novotny PJ, et al. Association of perceived medical errors with resident distress and empathy: a prospective longitudinal study. JAMA 2006;296:1071-8.

22. Mizrahi T. Managing medical mistakes: ideology, insularity and accountability among internists-in-training. Soc Sci Med 1984;19:135-46.

23. Sexton JB, Thomas EJ, Helmreich RL. Error, stress and teamwork in medicine and aviation: cross sectional surveys. BMJ 2000;320:745-9.

24. Wu AW, Folkman S, Mcphee SJ, et al. How house officers cope with their mistakes. West J Med 1993;159:565-9.

25. Tyssen R, Rovik JO, Valgum P, et al. Help-seeking for mental health problems among young physicians: is it the most ill that seeks help? A longitudinal and nationwide study. Soc Psychiatry Psychiatr Epidemiol 2004;39:989-93.

26. Baldwin PJ, Dodd M, Wrate RM. Young doctors' health II. Health and health behaviour. Soc Sci Med 1997;45:41-4.

27. Campbell S, Delva D. Physician do not heal thyself. Survey of personal health practices among medical residents. Can Fam Physician 2003;49:1121-7.

28. Farber NJ, Gilbert SG, Aboff BM, et al. Physicians' willingness to report impaired colleagues. Soc Sci Med 2005;61:1772-5.

29. Roberts LW, Warner TD, Rogers M, et al. Medical student ilness and impairment: a vignette-based survey study involving 955 students a 9 medical schools. Compr Psychiatry 2005;46:229-37.

30. Gabbe SG, Melville J, Mndel L, et al. Burnout in chairs of obstetrics and gynecology: diagnosis, treatment and prevention. Am J Obstet Gynecol 2002;186:601-12.

31. Warde CM, Moonesinghe K, Allen W, et al. Marital and parental satisfaction of married physicians with children. J Gen Intern Med 1999;14:157-65. 